\section{Method for selecting and enriching cells expressing low affinity ligands for cell surface receptors}

\author{
Hsi-Hsien Lin ${ }^{1,2}$, Martin Stacey², Gin-Wen Chang², John Q. Davies², \\ and Siamon Gordon ${ }^{2}$ \\ ${ }^{1}$ Chang Gung University, Taiwan, Republic of China and ${ }^{2}$ University of Oxford, \\ Oxford, UK
}

BioTechniques 38:696-698 (May 2005)
The affinity of protein-protein interaction in biological systems ranges from extremely strong, such as that between avidin and biotin $\left(\mathrm{K}_{\mathrm{a}}=\right.$ approximately $\left.10^{15} \mathrm{M}^{-1}\right)$ to the very weak, such as E-selectin and E-selectin ligand- $1\left(\mathrm{~K}_{\mathrm{a}}=\right.$ approximately $\left.7.4 \times 10^{4} \mathrm{M}^{-1} \mathrm{~S}^{-1}\right)(1)$. It is known that the interactions mediated by the surface receptors of immune cells are often of low-affinity and transient in nature (2). Despite weak affinities, cell surface interactions among immune cells have been shown to play an important role in many functions such as surveillance, immunosynapse formation, and activation (2). It is therefore desirable to devise a way to select and enrich cells expressing low-affinity ligands for specific immune cell surface receptors. Here we use the well-studied interaction between CD97 and CD55 as a model system to describe a simple method for this purpose.

Both CD97 and CD55 are cell surface proteins expressed predominantly on leukocytes (3-5). The binding of CD97 to CD55 has been demonstrated to be both of low affinity $(86 \mu \mathrm{M})$ and transient (off-rate; 0.6/s) (6). We and others have previously shown that the interaction is mediated exclusively by the epidermal growth factor (EGF)-like domains of CD97 and the short consensus repeats (SCR) of CD55 in a $\mathrm{Ca}^{2+}$-dependent fashion $(6,7)$. Furthermore, a CD97-like molecule, EMR2, has been shown not to bind CD55 even though it contains highly homologous EGF-like domains (97.5\% identity), therefore providing an ideal negative control (8).
Our method is based on (i) the efficient isolation and coupling of $\mathrm{Fc}$-fusion proteins to protein AGconjugated paramagnetic beads (Protein A/G Dynabeads ${ }^{\circledR}$; Dynal A.S., Oslo, Norway) (Figure 1A) and (ii) the simple capture and separation of ligand-positive cells from the Fcfusion protein-coated paramagnetic beads (Figure 1B). The coupling of the $\mathrm{Fc}$-fusion proteins to the protein AG-conjugated paramagnetic beads generates a multivalent protein probe, thereby increasing binding efficiency (Figure 1, A and B). As the major CD55-binding CD97 isoform contains three EGF-domains, we fused the three EGF-domains immediately upstream of the mouse $\mathrm{Fc}$ region (Figure 1A). CD97(EGF-1,2,5)-mFc and EMR2(EGF-1,2,5)-mFc fusion proteins were produced as described previously in transiently transfected HEK293T cells (Figure 1C).

Protein AG-conjugated paramagnetic beads were washed three times in binding buffer $[0.5 \%$ bovine serum albumin (BSA) in Hank's Balanced Salt solution (HBSS)], reconstituted in the binding buffer to the original concentration $(10 \mathrm{mg} / \mathrm{mL})$, and incubated at $4^{\circ} \mathrm{C}$ for $30 \mathrm{~min}$ with or without $2 \mu \mathrm{g} \mathrm{mFc}$-fusion protein $/ 10 \mu \mathrm{L}$ beads/reaction. Following the removal of unbound proteins by magnetic separation, the fusion protein-bead complexes were then resuspended in $20 \mu \mathrm{L}$ binding buffer/reaction. K562 cells $\left(\mathrm{CD} 55^{+}\right)$were washed, adjusted to a cell density of $3 \times 10^{6}$ cells $/ \mathrm{mL}$, and incubated at $4^{\circ} \mathrm{C}$ with the protein-bead

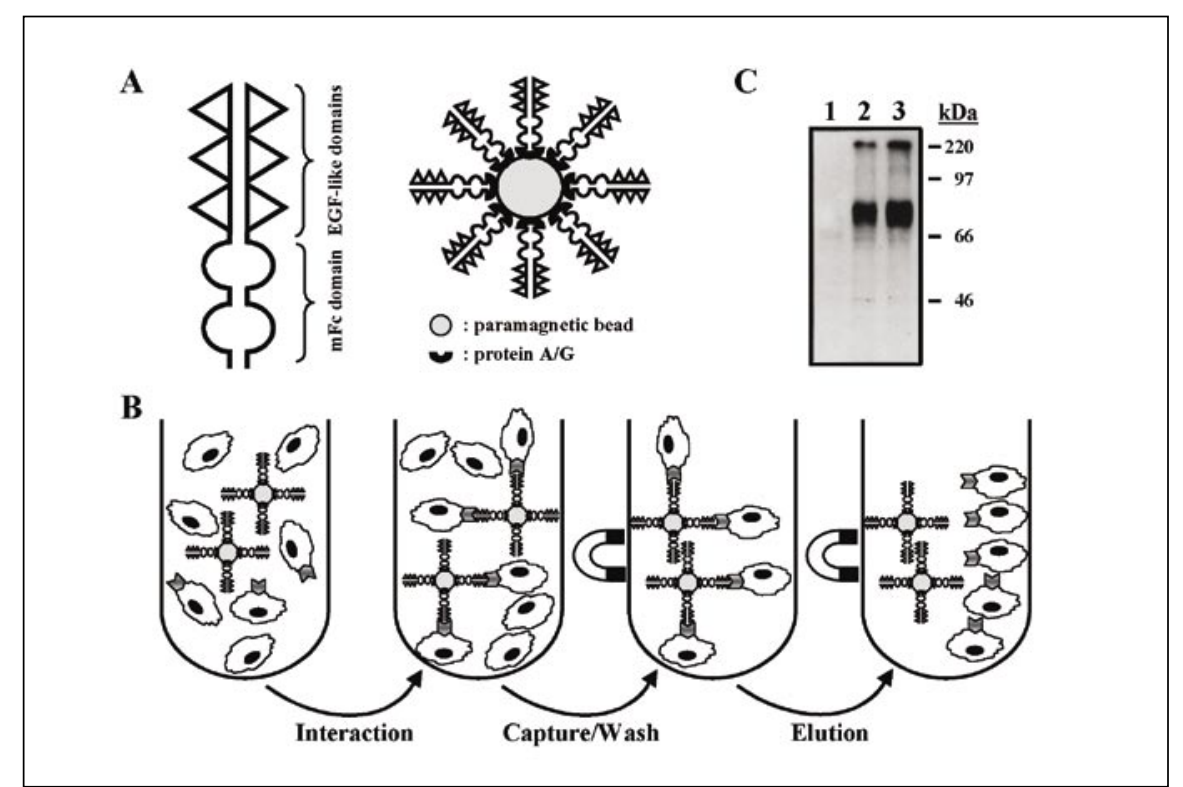

Figure 1. The selection of low-affinity ligand-bearing cells. (A) Representation of the $\mathrm{mFc}$-fusion proteins and the protein-bead complex. The epidermal growth factor (EGF)-like modules and the $\mathrm{mFc}$ region are represented by triangles and half-circles, respectively. The big gray circle represents protein AG-conjugated paramagnetic bead. The $\mathrm{mFc}$-fusion protein is shown as a dimer. (B) Diagrams depicting the selection and enrichment of ligand-bearing cells using the $\mathrm{mFc}$-fusion protein-bead complex. (C) Western blot analysis of CD97(1,2,5)-mFc and EMR2(1,2,5)-mFc fusion proteins. An Fc-specific antimouse immunoglobulin $\mathrm{G}$ horseradish peroxidase (IgG-HRP) was used to detect the $\mathrm{mFc}$-fusion proteins: lane 1, mock transfected conditioned medium; lane 2, CD97(1,2,5)-mFc; and lane 3, EMR2/1,2,5$\mathrm{mFc}$. The $\mathrm{mFc}$ fusion proteins were then purified and bound to the protein AG-conjugated paramagnetic bead to form the protein-bead probes. 


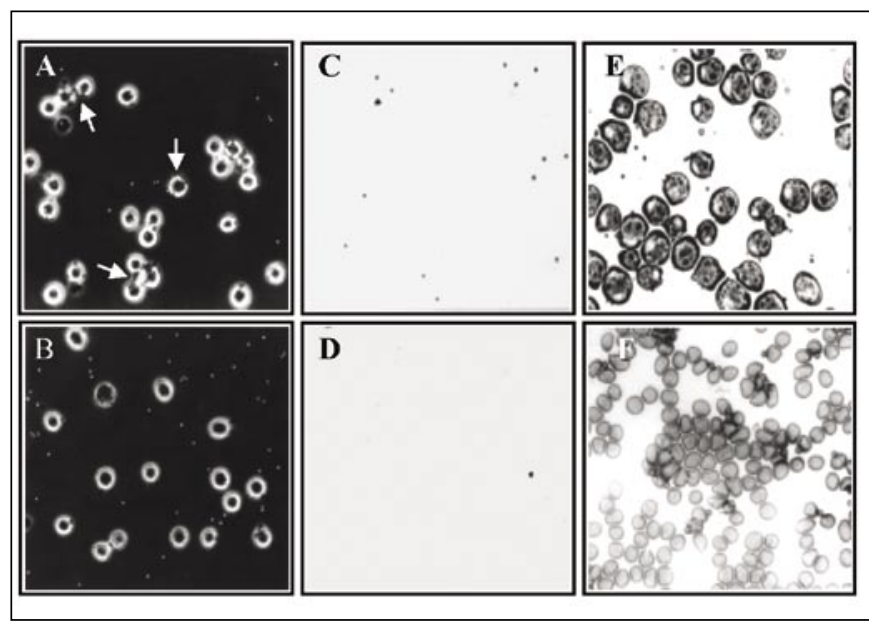

Figure 2. The binding and selection of CD55-bearing cells using CD97-beads CD55+-K562 cells were shown to be bound by CD97-beads (A, arrow) but not EMR2-beads (B). CD55 ${ }^{+}$-cells can be selected and enriched from CD97-beads ( $\mathrm{E}$ and $\mathrm{F}$ ) but not EMR2-beads ( $\mathrm{C}$ and $\mathrm{D}$ ), either from a homogenous cell population such as K562 cells (C and E) or from a mixed cell population such as blood (D and F).

complexes $(20 \mu \mathrm{L} / \mathrm{mL}$ cell solution/ reaction) for $1 \mathrm{~h}$ on an end-over-end rotator. When examined under the microscope, it was clear that K562 cells bound to CD97-bead but not EMR2bead complexes (Figure 2, A and B). The cell-bead mixture was then washed three times gently with the binding buffer, and the unbound cells were removed by magnetic separation. Beadbound cells were separated and isolated from the beads by incubation in binding buffer containing $5 \mathrm{mM}$ EDTA for 10 $\mathrm{min}$ at room temperature followed by magnetic separation (Figure 2, C and $\mathrm{E}$ ). The total number of recovered cells was determined by hemocytometer counting. We found that while CD97-coated beads bind and capture approximately $15 \%$ of input cells (approximately $4.5 \times 10^{5} / 3 \times 10^{6}$ ), control beads (EMR2-coated beads as well as beads alone) do not capture any significant number of cells at all (data not shown). Further studies have shown that the number of captured cells depends on the number of the input cells in a dose-dependent manner with the maximum capacity reaching approximately $1 \times 10^{7}$ cells $/ 10 \mu \mathrm{L}$ beads (data not shown). In addition, we have found that the protein-bead complexes can be reused several times with no apparent loss of binding efficiency (data not shown).
To test whether this method could also work in a mixed cell population, freshly obtained human whole blood was mixed with proteincoated beads. CD55-positive red blood cells were captured and isolated. Figure 2, D and $F$, show that a significantly pure population of red blood cells was obtained from CD97-coated beads, while no cells were purified by EMR2-coated beads. We further confirmed that the binding and isolation of red blood cells were indeed mediated by CD97CD55 interaction, as the addition of anti-CD55 antibody almost completely abolished the ability of CD97-coated beads to capture red blood cells (data not shown). Attempts to directly capture soluble CD55 ligands from K562 cell lysate using the CD97-coated beads were unsuccessful (data not shown). This is most likely due to the extremely weak and transient interaction between CD97 and CD55 and further supports the importance of multivalency in detecting low affinity receptor-ligand interaction.

In summary, we have described a method that allows the selection and enrichment of cells that express cellular ligands involved in the low-affinity interaction with specific cell surface receptors. This method provides an efficient way to produce multivalent protein probes with high binding efficiency, allowing for a simple procedure to select and enrich ligandbearing cells. This method is useful not only in characterizing receptor-ligand interaction, but also in providing an enriched source of ligand-bearing cells for downstream applications, such as the construction of expression libraries to identify unknown ligands.

\section{ACKNOWLEDGMENTS}

This study is supported by grants from the British Heart Foundation, Wellcome Trust, Medical Research Council, UK, and Chang Gung Memorial Hospital (CMRPD33008).

\section{COMPETING INTERESTS STATEMENT}

The authors declare no competing interests.

\section{REFERENCES}

1.Wild, M.K., M.C. Huang, U. Schulze-Horsel, P.A. van der Merwe, and D. Vestweber. 2001. Affinity, kinetics, and thermodynamics of E-selectin binding to E-selectin ligand-1. J. Biol. Chem. 276:31602-31612.

2.van der Merwe, P.A. and A.N. Barclay. 1994. Transient intercellular adhesion: the importance of weak protein-protein interactions. Trends Biochem. Sci. 19:354-358.

3.Hamann, J., W. Eichler, D. Hamann, H.M. Kerstens, P.J. Poddighe, J.M. Hoovers, E. Hartmann, M. Strauss, and R.A. van Lier. 1995. Expression cloning and chromosomal mapping of the leukocyte activation antigen CD97, a new seven-span transmembrane molecule of the secretion receptor superfamily with an unusual extracellular domain. J. Immunol. 155:1942-1950.

4.Hamann, J., B. Vogel, G.M. van Schijndel, and R.A. van Lier. 1996. The seven-span transmembrane receptor CD97 has a cellular ligand (CD55, DAF). J. Exp. Med. 184: 1185-1189.

5.Eichler, W., J. Hamann, and G. Aust. 1997. Expression characteristics of the human CD97 antigen. Tissue Antigens 50:429-438.

6.Lin, H.H., M. Stacey, C. Saxby, V. Knott, Y. Chaudhry, D. Evans, S. Gordon, A.J. McKnight, et al. 2001. Molecular analysis of the epidermal growth factor-like short consensus repeat domain-mediated protein-protein interactions: dissection of the CD97-CD55 complex. J. Biol. Chem. 276:24160-24169.

7.Hamann, J., C. Stortelers, E. Kiss-Toth, B. Vogel, W. Eichler, and R.A. van Lier. 1998. Characterization of the CD55 (DAF)-binding site on the seven-span transmembrane receptor CD97. Eur. J. Immunol. 28:1701-1707.

8.Lin, H.H., M. Stacey, J. Hamann, S. Gordon, and A.J. McKnight. 2000. Human EMR2, a novel EGF-TM7 molecule on chromosome $19 \mathrm{p} 13.1$, is closely related to CD97. Genomics 67:188-200.

Received 2 February 2005; accepted 22 February 2005.

Address correspondence to Hsi-Hsien Lin, Department of Microbiology and Immunology, Chang Gung University, 259 Wen-Hwa 1st Road, Tao-Yuan, Taiwan, Republic of China.e-mail: hhlin@mail.cgu.edu.tw 